\title{
LOCALIZING COMPACT BINARY INSPIRALS ON THE SKY USING GROUND-BASED GRAVITATIONAL WAVE INTERFEROMETERS
}

\author{
Samaya Nissanke ${ }^{1,2}$, Jonathan Sievers $^{3}$, Neal DalaL $^{3,4}$, and Daniel Holz $^{5,6}$ \\ ${ }^{1}$ JPL, California Institute of Technology, Pasadena, CA 91109, USA \\ 2 Theoretical Astrophysics, California Institute of Technology, Pasadena, CA 91125, USA \\ ${ }^{3}$ CITA, University of Toronto, Toronto, ON M5S 3H8, Canada \\ ${ }^{4}$ Department of Astronomy, University of Illinois, Urbana, IL 61801, USA \\ 5 Enrico Fermi Institute, Department of Physics, and Kavli Institute for \\ Cosmological Physics, University of Chicago, Chicago, IL 60637, USA \\ 6 Theoretical Division, Los Alamos National Laboratory, Los Alamos, NM 87545, USA \\ Received 2011 May 16; accepted 2011 July 7; published 2011 September 15
}

\begin{abstract}
The inspirals and mergers of compact binaries are among the most promising events for ground-based gravitationalwave $(\mathrm{GW})$ observatories. The detection of electromagnetic (EM) signals from these sources would provide complementary information to the GW signal. It is therefore important to determine the ability of GW detectors to localize compact binaries on the sky, so that they can be matched to their EM counterparts. We use Markov Chain Monte Carlo techniques to study sky localization using networks of ground-based interferometers. Using a coherentnetwork analysis, we find that the Laser Interferometer Gravitational Wave Observatory (LIGO)-Virgo network can localize $50 \%$ of their detected neutron star binaries to better than $50 \mathrm{deg}^{2}$ with a $95 \%$ confidence interval. The addition of the Large Scale Cryogenic Gravitational Wave Telescope (LCGT) and LIGO-Australia improves this to $12 \mathrm{deg}^{2}$. Using a more conservative coincident detection threshold, we find that $50 \%$ of detected neutron star binaries are localized to $13 \mathrm{deg}^{2}$ using the LIGO-Virgo network, and to $3 \mathrm{deg}^{2}$ using the LIGO-Virgo-LCGT-LIGOAustralia network. Our findings suggest that the coordination of GW observatories and EM facilities offers great promise.
\end{abstract}

Key words: gamma-ray burst: general - gravitational waves - stars: neutron - surveys

Online-only material: color figures

\section{INTRODUCTION}

The era of gravitational-wave (GW) astronomy is fast approaching. The advanced versions of the Laser Interferometer Gravitational Wave Observatory (LIGO; Barish \& Weiss 1999; Sigg \& the LIGO Scientific Collaboration 2008) and Virgo (Accadia et al. 2011) are expected to make their first detections within the coming decade. Furthermore, construction has begun on the Large Scale Cryogenic Gravitational Wave Telescope (LCGT; Kuroda \& the LCGT Collaboration 2010), and an additional advanced detector in Western Australia (referred to as LIGO-Australia or LAu in this work) is under serious consideration (Barriga et al. 2010; Munch et al. 2011). Inspiraling and merging compact-object binaries, composed of neutron stars (NS) and/or stellar-mass black holes (BH), are promising sources for these detectors. For an advanced LIGO-Virgo network, predicted event rates for NS-NS binaries range from 0.4 to 400 year $^{-1}$ (with 40 being the "realistic" number given in Abadie et al. 2010) detectable to distances of several hundred $\mathrm{Mpc}$, and approximately similar numbers apply for NS $-10 M_{\odot}$ BH binaries with detectable distances $>1$ Gpc. Nearly face-on merging NS binaries are considered likely progenitors of short hard $\gamma$-ray bursts (SHGRBs; e.g., Eichler et al. 1989). Metzger et al. (2010) suggest that NS binary mergers, irrespective of their orientation, may produce radioactive decay powered transients with absolute magnitude peak luminosities of $M_{V}=-15$ in the optical at $\sim$ day timescales. Thus, joint GW and electromagnetic (EM) observations can constrain the physics of generic NS binary mergers, the central engine and outflows of SHGRBs, and cosmological parameters (see Dalal et al. 2006; Nissanke et al. 2010, henceforth N10). GW events should, therefore, be localized on the sky to sufficient accuracy to enable a match with their EM counterparts. Henceforth, we use the terms "sky localization" to refer to the measured sky position and its associated error uncertainty, while "sky errors" applies only to area estimates.

For the typical NS binary inspiral event, whose duration is a few to tens of minutes in the detector frequency band, a single interferometer has a broad antenna pattern, and hence poor directional sensitivity. Two detectors restrict the sky localization to a single ring. In a network of three or more interferometers, relative arrival times of signals at each detector allow for the reconstruction of the source's sky location to within a generally elliptical error area of 1-100 $\mathrm{deg}^{2}$. For initial and advanced GW detector networks, previous studies (Sylvestre 2004; Cavalier et al. 2006; Blair et al. 2008; Fairhurst 2009; Klimenko et al. 2011; Schutz 2011) have explored sky errors for sources with both unmodeled ("burst") and modeled waveforms using analytically derived timing formulae and Fisher-matrix methods. At high signal-to-noise ratio (SNR $>100$ ) such methods are effective, providing lower bound error ellipses centered on the true values of the parameters of interest. However, the majority of sources for LIGO will be detected near threshold (SNR $\sim 8$ ). As discussed in N10 and Vallisneri (2008), at "low" SNR ( 20-100), parameter degeneracies can lead to quantitative and qualitative errors in the Fisher-matrix approximation of the posterior probability distribution functions (PDFs) for source parameters. Since early GW detections will likely be low-SNR, a full treatment of the sky localization will be critical when matching the GW event to an EM counterpart both (1) to ensure high-probability regions are not falsely excluded from consideration and (2) to minimize the number of 
false-positive transients that must be dealt with. Later, should event rates reach tens per year as predicted, the ease of finding counterparts will be directly related to how well GW networks can localize events. In this paper we use Markov Chain Monte Carlo (MCMC) techniques to map the full PDF of the sky location where an event may have occurred. Specifically, we examine sky localization for non-spinning NS-NS populations for advanced GW detector networks, including LIGO (the two $4 \mathrm{~km}$ sites situated at Hanford and Livingston), Virgo, LCGT, and LIGO-Australia. For conciseness, we restrict our analysis here to NS-NS binaries, results for NS-BH binaries being similar. For networks comprising the initial versions of LIGO and Virgo detectors, several works (e.g., Röver et al. 2007; van der Sluys et al. 2009; Raymond et al. 2009) have addressed sky localization using MCMC techniques for single NS-NS and spin-precessing NS-BH systems. After the present article was submitted for review, a similar MCMC study was performed by Aylott et al. (2011). They focused on the addition of LIGO Australia, and found results consistent with ours.

We follow techniques similar to those developed in N10, which focuses on distance determination using advanced GW detector networks for sources with known EM counterparts. In contrast to N10, here we instead ask how well a network of ground-based GW detectors can determine the sky positions of previously unknown compact binary sources, a central question when planning complementary EM observations for counterparts.

This paper is organized as follows. Section 2 outlines the extraction of sky position from the GWs emitted by inspiraling binaries. Section 3 discusses our sky localization results for individual systems and populations of NS-NS binaries. Section 4 presents our conclusions.

\section{SKY LOCALIZATION DETERMINATION}

Based on optimal matched filtering (Oppenheim et al. 1983; Finn 1992; Cutler \& Flanagan 1994), we extract the sky position n for each NS-NS binary using knowledge of the expected GW waveform, where $\mathbf{n} \equiv(\theta, \phi)$ is the vector pointing to a binary on the sky (the waves therefore propagate to the Earth along $-\mathbf{n}$ ). The colatitude $\theta$ and longitude $\phi$ are related to the declination $\delta$ and right ascension $\alpha$, by $\theta=\pi / 2-\delta$ and $\phi=\alpha$-GAST respectively, where GAST is Greenwich Apparent Sidereal Time. We use only the early inspiral portion of the waveform, which for low-mass systems provides most of the signal for advanced detectors (Flanagan \& Hughes 1998) and is modeled accurately using post-Newtonian (PN) expansions in general relativity. Specifically, we use the non-spinning restricted $2 \mathrm{PN}$ waveform in the frequency domain for the two GW polarizations $h_{+}$and $h_{\times}$; see Equations (12)-(14) in N10. The detector antenna functions depend on $\mathbf{n}$ and the binary's polarization angle. The overall amplitude of the GW waveform encodes the source's orientation, sky location, luminosity distance, and redshifted chirp mass (see discussion in N10). For simplicity we assume that the inspiral waveform ends abruptly prior to merger at the innermost stable circular orbit. The time of flight from source at direction $\mathbf{n}$ to detector at location $\mathbf{r}$ involves the scalar product $\mathbf{n} \cdot \mathbf{r}$, and differences in time-of-flight among detectors in the network dominate sky localization.

In order to infer the sky position $(\cos \theta, \phi)$, we explicitly map out the posterior PDF for all source parameters (including chirp mass, orientation, etc.) given an observed data stream at a detector, using MCMC. The Metropolis-Hastings MCMC algorithm used is based on a generic version of CosmoMC, described in Lewis \& Bridle (2002). For simplicity, we assume Gaussian, stationary, and zero-mean noise that is independent and uncorrelated between detectors. We take the anticipated noise sensitivity curve for a single advanced LIGO detector, given in Harry \& the LIGO Scientific Collaboration (2010), for broadband tuning, to be representative of all our detectors, imposing a low-frequency cutoff of $10 \mathrm{~Hz}$. Since we trigger detections off the expected and not the observed SNR, we do not incorporate selection effects into our analysis. Therefore, we take prior distributions in all source parameters to be flat over the region of sample space where the binary is detectable at an expected network SNR $=3.5$. The expected network SNR is defined as the root sum square of the expected individual detector SNRs. For each MCMC simulation, we derive solid angle areas over $(\cos \theta, \phi)$ for $68 \%, 95 \%$, and $99 \%$ confidence regions.

\subsection{Detected Binary Populations}

The selection of our "detected" binaries is central to the derivation of representative sky localization statistics for binary populations (Schutz 2011). We simulate a million binaries out to $z=1$, assuming a constant comoving volume density in a $\Lambda$ CDM universe (Komatsu et al. 2009), and with random binary sky positions and orientations. Each NS has a physical mass of $1.4 M_{\odot}$. We consider two plausible scenarios where each binary in the total population is selected: (1) if its expected network SNR is greater than a network threshold of 8.5 (Case I) and (2) if each expected SNR at both LIGO Hanford and LIGO Livingston has a value of 6 or higher and if the expected network SNR is above 12 (Case II). We choose to threshold off the LIGO detectors, as opposed to any other two detectors, only as a representative example out of all other possible coincident threshold scenarios. Case I uses a network thresholding criterion similar to that used in N10 and Schutz (2011).

\section{RESULTS AND DISCUSSION}

\subsection{Individual Binaries}

We first examine sky localization for NS-NS binaries taken at random from our Case I or Case II detected samples. For each binary we assign a unique noise realization to each detector, which we keep constant when adding and subtracting detectors to a network. This enables a meaningful comparison between the performance of different networks. We consider networks comprising combinations of LIGO, Virgo, LAu, and LCGT.

In agreement with Fairhurst (2011) and Wen \& Chen (2010), we find elliptically shaped errors for the majority of our examined NS-NS binaries. This is unsurprising given that sky localization reconstruction is dominated by differing $\mathrm{GW}$ arrival times at each detector rather than the direction-dependent antenna functions in the GW amplitude. For the three-element LIGO-Virgo network, sources located toward the detector plane produce elongated error ellipses and have relatively poor angular resolution. However, the error ellipse significantly decreases in size as additional detectors are added to the network. The inclusion of LAu is particularly favorable and breaks the LIGO-Virgo(-LCGT) degenerate plane. As a representative example, Figure 1 shows a relatively high-SNR GW signal for a NS-NS binary located at $180 \mathrm{Mpc}$ with an inclination angle of $\cos \iota=0.7$ and a sky location of $(\cos \theta=-0.3, \phi=2.9)$. The expected SNRs at LIGO Hanford, LIGO Livingston, Virgo, LAu, and LCGT are 6.7, 7.8, 12.4, 10.5, and 8.9, respectively. 

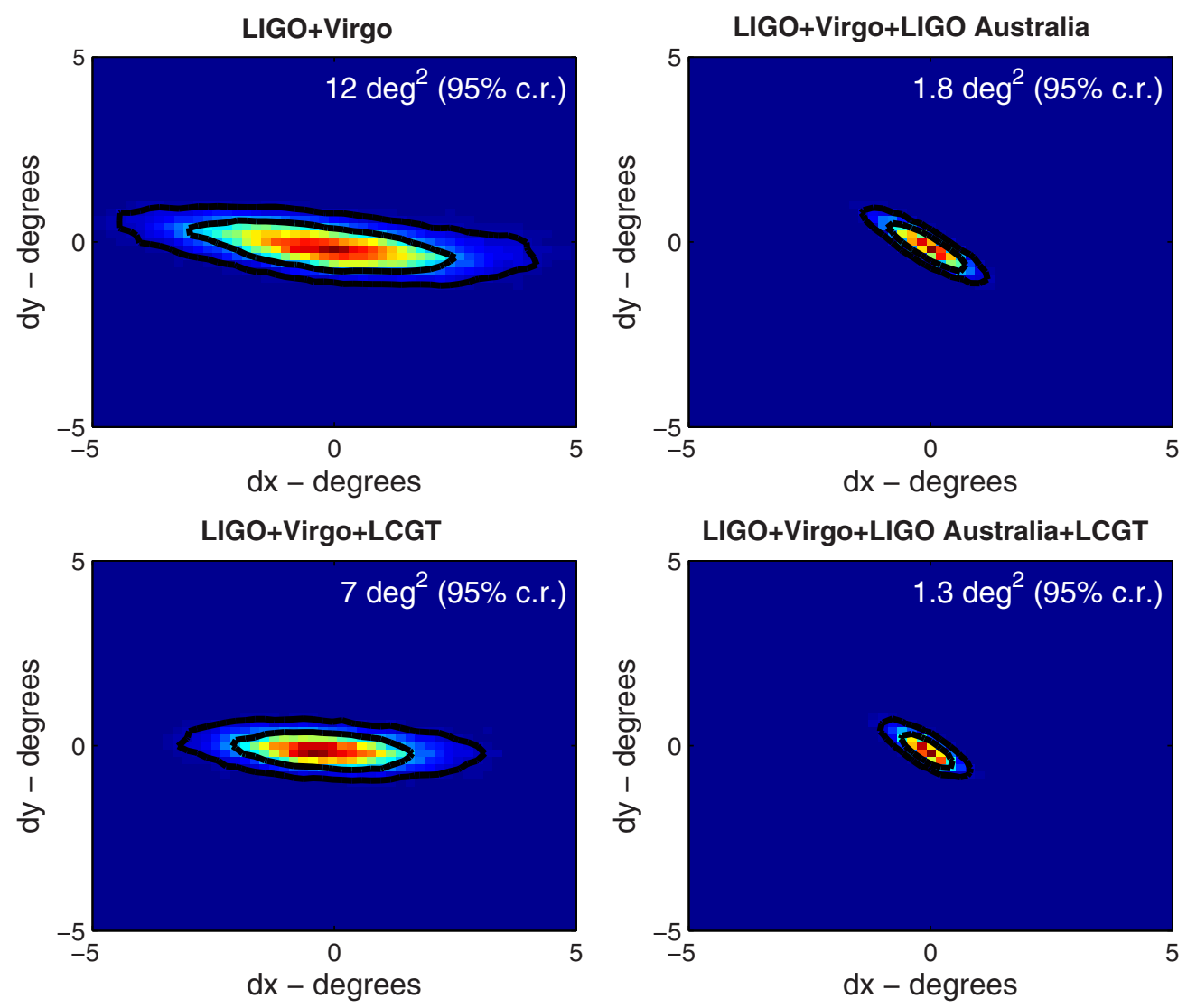

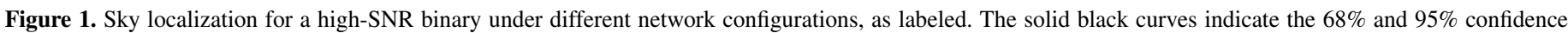

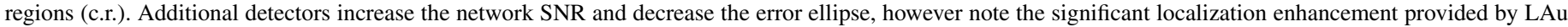
in particular. The origin $(0,0)$ of each plot represents the source's true position, and the solid black lines denote the confidence regions.

(A color version of this figure is available in the online journal.)

The specific orientation and shape of the ellipse are dependent upon the sky position and orientation, and noise realization.

However, for a handful of events near threshold, we find that sky error areas are considerably non-ellipsoidal and exhibit multimodal distributions, in particular with networks that do not include LAu. As expected, their errors are not centered on the source's true sky position and we find that $5 \%$ of our selected binaries have true positions that lie outside the $95 \%$ confidence region. Analyses such as Fairhurst (2011), Wen \& Chen (2010), and Schutz (2011) cannot reproduce such features due to limiting assumptions implicit in timing and Fisher information methods. Figure 2 shows an example of a low-SNR NS-NS binary located at $567 \mathrm{Mpc}$ with an inclination angle of $\cos \iota=-0.93$ and a sky location of $(\cos \theta=-0.36, \phi=1.5)$. The expected SNRs at LIGO Hanford, LIGO Livingston, Virgo, LAu, and LCGT are 5.4, 6.2, 3.1, 5.6, and 3.0, respectively.

We find that error areas typically decrease by a factor of 4-5 by including LAu in the network. For particularly weak signals, multimodal peaks and statistical biases occur because multiple likelihood peaks for different values of the GW waveform's amplitude become indistinguishable from each other due to the uncertainties in the signal's time of arrival at each detector.

\subsection{Detected Samples of Binaries}

We now examine cumulative distribution in sky errors for ensembles of GW NS-NS events using different detector networks. We randomly choose events from our samples of detected NS-NS binaries using the two different selection criteria detailed in Section 2.1.

\subsubsection{Case I: Triggering on a Network of Detectors}

In Case I we set a total GW detector network threshold of 8.5, which implies an approximate SNR threshold per detector of $8.5 / \sqrt{5} \sim 3.8$ for each of the five detectors. As a first approximation, we estimate a detectable range of events within the maximum network capability: each detector in the fivedetector network has a "weighted geometric average" (optimal) range of about $420 \mathrm{Mpc}(940 \mathrm{Mpc})$ for NS-NS events. ${ }^{7}$ The geometric average statistic used here is a weighted angular average over all sky positions and orientations, which is a factor of $\sim 2.24$ smaller than the optimal range for a face-on binary that is located directly above the detector (see Finn \& Chernoff 1993).

In order to obtain detection event rates, we follow the approach given in Abadie et al. (2010) where detection ranges are derived by thresholding off a single LIGO SNR of 8. As the noise in reality is non-Gaussian and non-stationary, Abadie et al. (2010) use the range for a single detector to represent the network of LIGO-Virgo detectors in order to achieve desired false alarm rates. For comparison purposes, we apply the same argument to our analysis: a five-detector network will have an approximate threshold per detector of $(8.5 \times \sqrt{3}) / \sqrt{5} \sim 6.6$, and hence a geometric average (optimal) range of about $240 \mathrm{Mpc}$ (540 Mpc) for NS-NS events. Abadie et al. (2010) provide a simple prescription to compute the GW NS-NS detection rate using

\footnotetext{
7 Similar to Abadie et al. (2010), we do not henceforth incorporate cosmological redshifts for our NS masses when estimating detectable ranges and rates.
} 

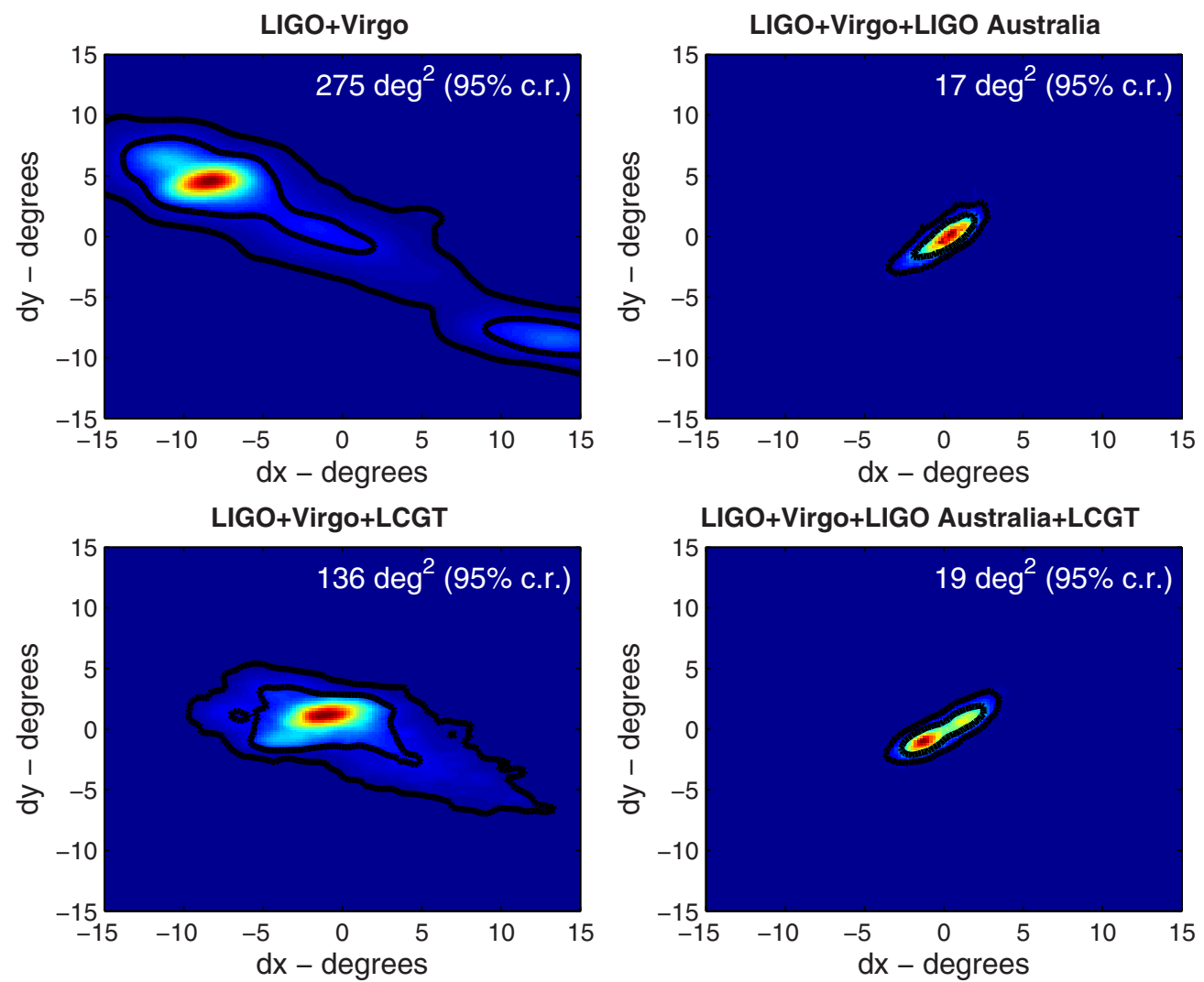

Figure 2. Sky localization for a low-SNR binary. Contour levels are as in Figure 1. In this example, the three-detector network finds multiple, widely separated islands of high likelihood, offset from the true location. Additional detectors, however, can break underlying degeneracies. Furthermore, the binary's sky error at $95 \%$ c.r increases slightly with the five-detector network compared to the four-detector (LAu) network, because in this particular instance, the noise realization is unfavorable at LCGT.

(A color version of this figure is available in the online journal.)

NS-NS coalescence rates per galaxy, and the number of galaxies accessible within a given GW reach. The NS-NS coalescence rate per galaxy is estimated either by extrapolating from the observed sample of NS-NS binaries detected via pulsar measurements or by using population-synthesis methods. Using Table II with the associated low-realistic-high-maximum NS-NS coalescence rates per galaxy ${ }^{8}$ and Equations (1) and (5) in Abadie et al. (2010), we define our realistic detection rate of $\sim 65$ NS-NS binaries per year. Corresponding low, high, and maximum detection rates are, respectively, 0.7, $\sim 660$, and $\sim 2650$ NS-NS binaries per year seen by the LIGO+Virgo+LAu+LCGT network.

Turning to our results, we take a sample of 98 NS-NS binaries detected by the full five-detector network, corresponding to an observation time of $\sim 8$ months based on our realistic rate estimates. In Figure 3, we show the normalized cumulative distribution of sky errors in square degrees for our sample. We show distributions for subsets of systems detected by different networks which are normalized to the full sample illustrating the reduced number of detections. Table 1 illustrates sky errors for $25 \%, 50 \%$, and $75 \%$ of NS-NS binaries from the sample detected by a particular network. Among notable features: (1) the addition of detectors to the network, in particular LAu, significantly reduces sky localization errors. We find that $50 \%$ of all detectable NS- NSs are localized at $95 \%$ confidence

\footnotetext{
Abadie et al. (2010) assign rate estimates to one of four categories as detailed in their Section IV; when rate PDFs are available, realistic refers to the mean of the PDF, low and high denote the $95 \%$ pessimistic and optimistic confidence intervals, and maximum is the upper limit quoted in published literature.
}

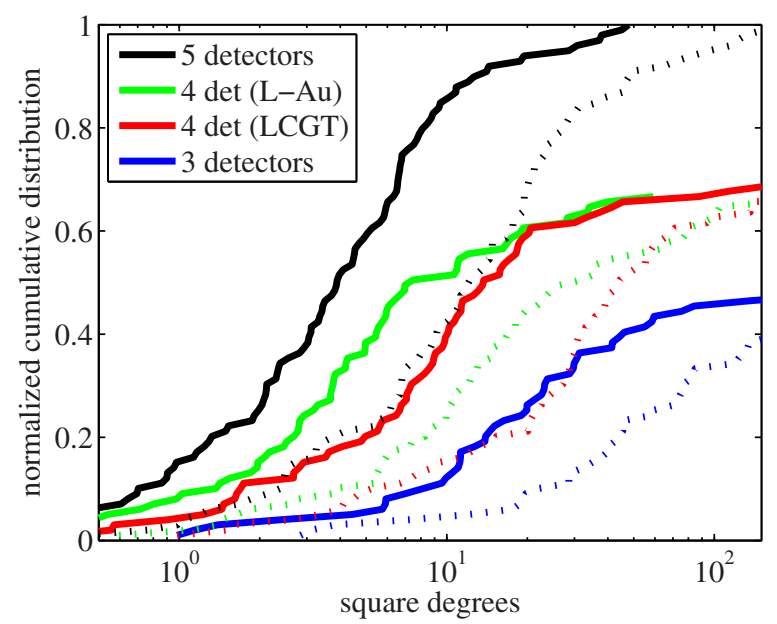

Figure 3. Normalized cumulative distributions as a function of the sky-error area (square degrees) of a sample of NS-NS binaries in Case I detection scenario. Key: solid/dotted lines denote $68 \%$ and $95 \%$ c.r., respectively. Black: LIGO+Virgo+LAu+LCGT network, green: LIGO+Virgo+LAu, red: LIGO+Virgo+LCGT, and blue: LIGO+Virgo only.

(A color version of this figure is available in the online journal.)

region to within $10-20 \mathrm{deg}^{2}$ with any four- or five-detector network including $\mathrm{LAu}$, and to within $110 \mathrm{deg}^{2}$ with only the three LIGO+Virgo network. (2) The number of detected binaries doubles as the number of detectors in a network increases from three to five. 
Table 1

Sky Errors in $\mathrm{deg}^{2}$ at 68\%/95\%/99\% Confidence Regions (c.r.) for NS-NS Binary Populations Detected Using Two Different Selection Criteria with Varying Networks

\begin{tabular}{lccccc}
\hline \hline Network & Fraction of Binaries & LIGO+Virgo (LLV) & LLV+LAu & LLV+LCGT & LLV+LAu+LCGT \\
\hline & & $\begin{array}{c}68 \% / 95 \% / 99 \% \\
\text { in } \text { deg }^{2}\end{array}$ & $\begin{array}{c}68 \% / 95 \% / 99 \% \\
\text { in } \operatorname{deg}^{2}\end{array}$ & $\begin{array}{c}68 \% / 95 \% / 99 \% \\
\text { in deg }^{2}\end{array}$ & $\begin{array}{c}68 \% / 95 \% / 99 \% \\
\text { in } \text { deg }^{2}\end{array}$ \\
\hline Case I & $25 \%$ of binaries & $11 / 29 / 45$ & $2 / 5 / 10$ & $4 / 12 / 20$ & $2 / 7 / 10$ \\
& Median percentile & $17 / 53 / 88$ & $4 / 13 / 22$ & $9 / 30 / 49$ & $4 / 12 / 20$ \\
& $75 \%$ of binaries & $30 / 124 / 200$ & $7 / 30 / 53$ & $15 / 47 / 82$ & $7 / 22 / 39$ \\
\hline Case II & $25 \%$ of binaries & $2 / 9 / 15$ & $1 / 2 / 3$ & $1 / 3 / 5$ & $1 / 2 / 3$ \\
& Median percentile & $5 / 13 / 21$ & $2 / 5 / 9$ & $3 / 7 / 11$ & $1 / 3 / 5$ \\
& $75 \%$ of binaries & $12 / 36 / 60$ & $3 / 9 / 14$ & $5 / 20 / 33$ & $2 / 7 / 12$ \\
\hline Case I & $\%$ of detected binaries & 49 & 66 & 69 & 100 \\
\hline Case II & $\%$ of detected binaries & 73 & 97 & 83 & 100
\end{tabular}

Note. Percentages of detected binaries as a function of detector network are also indicated.

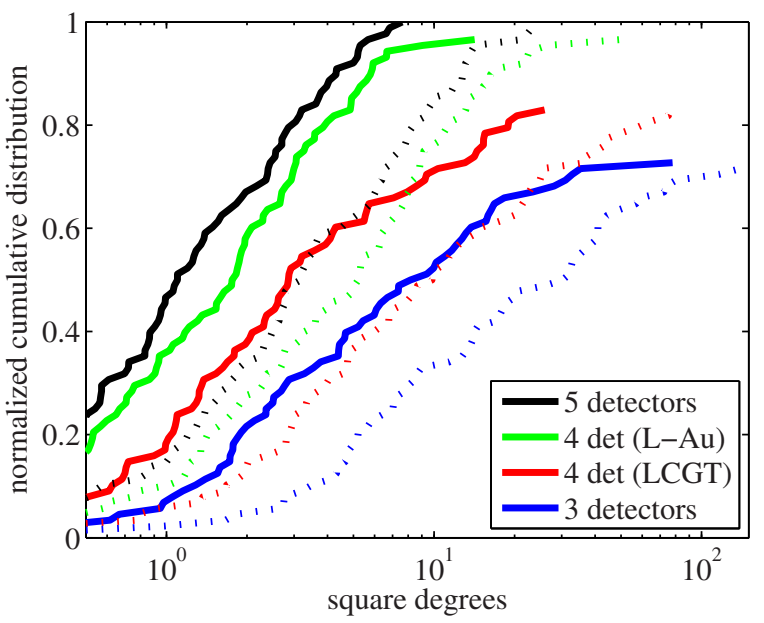

Figure 4. Normalized cumulative distributions of sky-error area (square degrees) for a sample of NS-NS binaries in Case II detection scenario. The NS-NS detection rate in Case II is approximately four times less than that in Case I. Key: solid/dotted lines denote $68 \%$ and $95 \%$ c.r., respectively. Black: LIGO+Virgo+LAu+LCGT network, green: LIGO+Virgo+LAu, red: LIGO+Virgo+LCGT, and blue: LIGO+Virgo only.

(A color version of this figure is available in the online journal.)

\subsubsection{Case II: Triggering on Individual LIGO Detectors}

For the Case II scenario we define detection using the more stringent requirement of SNR $>6$ at each LIGO detector. Using an SNR of $10.4(\sim 6 \times \sqrt{3})$ in each LIGO detector (where the factor of $\sqrt{3}$ follows from the Abadie et al. (2010) correction for non-stationary and non-Gaussian noise), we compute a weighted geometric average (optimal) range of $150 \mathrm{Mpc}$ (340 Mpc). Invoking a similar argument as in Case I, we estimate a realistic detection rate of $\sim 17$ NS-NS binaries per year. Corresponding low, high, and maximum detection rates are, respectively, 0.2 , $\sim 165$, and $\sim 660$ NS-NS binaries per year.

Similar to Case I, we take a detected sample of 88 NS-NS binaries seen by the full LIGO-Virgo-LAu-LCGT network. Figure 4 shows the normalized cumulative distribution of our NS-NS binary sample as a function of sky error in square degrees. We find that $50 \%$ of NS-NSs are detected at $95 \%$ confidence level to within $5 \mathrm{deg}^{2}$ with any network including $\mathrm{LAu}$, and $\sim 15 \mathrm{deg}^{2}$ with only the LIGO+Virgo network. As before, Table 1 shows the cumulative distribution of sky errors for NS-NS binaries detected by different networks. Despite differences in our analyses and astrophysical population models used, our results are consistent with Fairhurst (2011) and Schutz (2011). Once again, the inclusion of LAu substantially improves localization errors and the binary detection rate rises with more detectors. In contrast to Case I, however, we find that the number of detected binaries increases only by a factor of 1.3 going from a three- to five-detector network, because both LIGOs must have SNRs $>6$. Using all five detectors, the Case II scenario results in a factor of $\sim 5$ fewer binaries than in the Case I counterpart. We also find that the addition of a fourth or a fifth detector does not improve sky coverage as significantly in Case II as in Case I.

\section{CONCLUSIONS}

This work examines sky localization for both individual systems and populations of NS-NS binaries using different networks of advanced GW detectors. For the majority of optimally oriented NS-NS binaries examined, we show good agreement of our MCMC derived errors with the error ellipses obtained from analytical timing accuracy and Fisher-matrix formulae. However, for a handful of SNR signals at threshold (in particular with the standard geometrically degenerate LIGO-Virgo network), we show that sky error regions can be non-ellipsoidal and non-contiguous, show multimodal distributions, and the best fits can be shifted away from their true values. Of particular relevance, our results show that the inclusion of LIGO-Australia in a worldwide GW detector network improves localization errors both for individual and populations of binaries up to a factor of $\sim 5$, reducing the appearance of multimodal islands. Finally, the number of detected binaries increases with the number of detectors in a network.

A natural extension of this paper is to include astrophysically realistic populations of NS-NS and spin-precessing NS-BH binaries. Outstanding questions that are yet to be addressed are the implications of our findings for observational EM follow up. Our results show that measurements of astrophysical populations of $\mathrm{GW}$ events result in error areas of $\sim 10 \mathrm{deg}^{2}$. In the optical, cross-correlating localization error areas $>10 \mathrm{deg}^{2}$ with as complete as possible galaxy catalogs, such as the "Local Universe" census proposed by Kulkarni \& Kasliwal (2009), should aid EM search strategies (M. Kasliwal et al. 2011, in preparation). As recent observations indicate, a small number ( five at present) of SHGRBs appear to be located several tens of kpc away from their host galaxies (e.g., Berger 2010; 
Fong et al. 2010). Thus, search strategies will need to include the possibility of such effects when looking for transients of NS binary mergers. Moreover, time-domain surveys provide an estimate of the false-positive rate of dynamic galactic (foreground) and extragalactic (background) transients. Currently, operating and future optical and radio EM facilities are thus capable of preparing for and performing follow-up of GW events.

We are grateful to Josh Bloom, Scott Hughes, Sterl Phinney, Bangalore Sathyaprakash, and Michele Vallisneri for suggestions in the early development of the work. We thank Chad Galley, Mansi Kasliwal, Ilya Mandel, and Eran Ofek for careful reading of the manuscript, and Yanbei Chen, Curt Cutler, Wen-Fai Fong, Chris Hirata, Tom Prince, Bernard Schutz, Kip Thorne, Linqing Wen, and Stan Whitcomb for helpful discussions. Simulations were performed using the Sunnyvale cluster at CITA, which is funded by NSERC and CIAR. S.M.N.'s research was carried out at the Jet Propulsion Laboratory, California Institute of Technology under a contract with National Aeronautics and Space Administration.

\section{REFERENCES}

Abadie, J., Abbott, B. P., Abbott, R., et al. 2010, Class. Quantum Grav., 27, 173001

Accadia, T., Acernese, F., Antonucci, F., et al. 2011, Class. Quantum Grav., 28, 114002

Aylott, B., Farr, B., Kalogera, V., et al. 2011, arXiv:1106.2547

Barish, B. C., \& Weiss, R. 1999, Phys. Today, 52, 44

Barriga, P., Blair, D. G., Coward, D., et al. 2010, Class. Quantum Grav., 27, 084005

Berger, E. 2010, ApJ, 722, 1946

Blair, D. G., Barriga, P., Brooks, A. F., et al. 2008, J. Phys. Conf. Ser., 122, 012001
Cavalier, F., Barsuglia, M., Bizouard, M.-A., et al. 2006, Phys. Rev. D, 74, 082004

Cutler, C., \& Flanagan, É. E. 1994, Phys. Rev. D, 49, 2658

Dalal, N., Holz, D. E., Hughes, S. A., \& Jain, B. 2006, Phys. Rev. D, 74, 063006

Eichler, D., Livio, M., Piran, T., \& Schramm, D. N. 1989, Nature, 340, 126

Fairhurst, S. 2009, New J. Phys., 11, 123006

Fairhurst, S. 2011, Class. Quantum Grav., 28, 105021

Finn, L. S. 1992, Phys. Rev. D, 46, 5236

Finn, L. S., \& Chernoff, D. F. 1993, Phys. Rev. D, 47, 2198

Flanagan, É. É., \& Hughes, S. A. 1998, Phys. Rev. D, 57, 4535

Fong, W., Berger, E., \& Fox, D. B. 2010, ApJ, 708, 1

Harry, G. M., \& the LIGO Scientific Collaboration 2010, Class. Quantum Grav., 27, 084006

Klimenko, S., Vedovato, G., Drago, M., et al. 2011, Phys. Rev. D, 83, 102001

Komatsu, E., Dunkley, J., Nolta, M. R., et al. 2009, ApJS, 180, 330

Kulkarni, S., \& Kasliwal, M. M. 2009, in Proc. RIKEN Symp., Astrophysics with All-Sky X-Ray Observations, 312 (arXiv:0903.0218)

Kuroda, K., \& the LCGT Collaboration 2010, Class. Quantum Grav., 27, 084004

Lewis, A., \& Bridle, S. 2002, Phys. Rev. D, 66, 103511

Metzger, B. D., Martínez-Pinedo, G., Darbha, S., et al. 2010, MNRAS, 406, 2650

Munch, J., Marx, J., Whitcomb, S., et al. 2011, https://dcc.ligo.org/cgi-bin/ DocDB/ShowDocument?docid $=11666$

Nissanke, S., Holz, D. E., Hughes, S. A., Dalal, N., \& Sievers, J. L. 2010, ApJ, 725, 496 (N10)

Oppenheim, A. V., Willsky, A. S., \& Young, I. T. 1983, Signals and Systems (Englewood Cliffs, NJ: Prentice-Hall)

Raymond, V., van der Sluys, M. V., Mandel, I., et al. 2009, Class. Quantum Grav., 26, 114007

Röver, C., Meyer, R., \& Christensen, N. 2007, Phys. Rev. D, 75, 062004

Schutz, B. F. 2011, Class. Quantum Grav., 28, 125023

Sigg, D., \& the LIGO Scientific Collaboration 2008, Class. Quantum Grav., 25, 114041

Sylvestre, J. 2004, Class. Quantum Grav., 21, 775

Vallisneri, M. 2008, Phys. Rev. D, 77, 042001

van der Sluys, M., Mandel, I., Raymond, V., et al. 2009, Class. Quantum Grav., 26, 204010

Wen, L., \& Chen, Y. 2010, Phys. Rev. D, 81, 082001 